\title{
System Reliability Models with Stress Covariates for Changing Load Profiles
}

\author{
Akira Hada, Rutgers University \\ David Coit, Rutgers University \\ Mark Agnello, U.S. Navy \\ Keith Megow, U.S. Navy
}

Key Words: Simulation Models, Failure Rate Model, Accelerated Life

\section{SUMMARY \& CONCLUSIONS}

This paper presents various models to estimate reliability for a future profile with increased stress using the current observations to develop a model for future reliability. For the foreseeable future the equipment itself will not change, and thus the increase of the load or stress, that the system is exposed to, may decrease reliability and increase maintenance. The model is required to determine the impact of these future profiles. This paper is motivated by an actual application modeling the current and anticipated future reliability of naval aircraft launch and recovery equipment (ALRE). The cyclesto-failure data (CTF) was generated by simulation and serves as the basis in explaining four methods that will be evaluated during 2011-2012.

System reliability models are presented and demonstrated to anticipate system reliability and availability for changing and increasing applied loading distributions. These models are intended for systems whose components are exposed to predictable and quantifiable, but changing loading patterns. In the intended application, the models will be used to estimate the future reliability of naval aircraft catapult and arresting gear when subjected to different air wing compositions. Each air wing usage profile is potentially different, forming a distribution of usage stresses, and this distribution is shifting with time, as aircraft weight and missions change. The current ALRE systems will continue to be used for the foreseeable future and the model is required to predict future performance and to identify the most unreliable components or problems within the existing design.

Weibull distribution models are used in the typical fashion to model component failure times, but the initial Weibull distribution parameters are mathematical functions of the current, known applied stress distributions. These component models are then used within a discrete event simulation model to predict system reliability and availability. After this initial evaluation, models and software normally applied for accelerated life testing applications are used to develop models which have Weibull distribution parameters that are both mathematical functions with usage stress covariates and also mathematical functions of the distribution or variations in the changing applied loading conditions. Four specific modeling approaches are presented, and compared and contrasted based on data requirements, practicality and other criteria. The four modeling approaches involved models and data analysis at the component-level based on (i) linear stress adjusted usage measures in place of time, (ii) nonlinear stress adjusted usage measures in place of time, (iii) Weibull shape parameters modeled using a general log-linear model based on the mean and standard deviation of critical stress measures in a changing environment, and (iv) Weibull shape parameters modeled using a general log-linear model based on the distributional form of critical stress measures in a changing environment. The component models are then assembled into a system model. This methodology is demonstrated and compared on an example system using simulated data. The models collectively provide a practical methodology to use existing failure and usage stress data to predict future reliability based on a changing and increasing loading pattern.

$$
\begin{aligned}
& \text { ACROYNYM AND EQUATION NOTATION } \\
& \text { CTF }=\text { Cycle Time Failure } \\
& \text { MLE }=\text { Maximum Likelihood Estimator } \\
& t_{\mathrm{s}} \quad=\text { total number of stress cycles } \\
& t_{\mathrm{i}}=\text { total number of stress cycles of the } i^{\text {th }} \text { type } \\
& s_{i}=\text { stress for the } i^{\text {th }} \text { stress type } \\
& s_{\mathrm{O}} \quad=\text { baseline stress } \\
& a_{j}=\text { nonlinear adjustment factor for component } j \\
& f(t)=\text { probability density function for cycles-to-failure } \\
& \beta \quad=\text { Weibull shape parameter } \\
& \eta \quad=\text { Weibull scale parameter } \\
& \eta_{\mathrm{o}} \quad=\text { baseline Weibull scale parameter } \\
& t \quad=\text { number of usage cycles } \\
& \mu \quad=\text { average stress } \\
& \sigma \quad=\text { standard deviation of stresses } \\
& b_{1} \quad=\text { parameter coefficient for mean stress }
\end{aligned}
$$


$b_{2}=$ parameter coefficient for standard deviation

$x_{1}=1^{\text {st }}$ relative stress frequency

$x_{2}=2^{\text {nd }}$ relative stress frequency

$x_{3}=3^{\text {rd }}$ relative stress frequency

\section{INTRODUCTION}

The goal of this paper is to model a discrete cyclic loading system with predictable, quantifiable and changing loading patterns. Every usage cycle can be different, due to a different load, pressure or force being applied to the system. Taking this cyclic data and forming a stress distribution only describes past occurrences. However, the future loads on the system are anticipated to be increasing due to changing usage profiles. This creates a shifting stress distribution with time. An example of this shift is depicted in Figure 1.

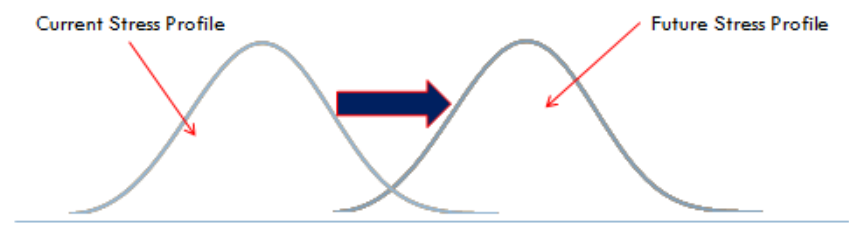

Figure 1: Movement of the current stress profile to the future stress profile with time

In this analysis, the existing system is analyzed considering the future stresses. A simulation model is then used to predict the system's performance and identify the most unreliable components given the changing stresses associated with the new usage profile. Examples of a discrete loading system would be a hydraulic crane that continually lifts different types of weights or a washing machine or planes landing on a runway. The discrete loads determine the stresses and a cycle must be clearly defined. Once the system and cycle are identified, the effects of the future stress must be determined. An example of this problem would be if a crane, which continually lifts $200 \mathrm{lbs}, 400 \mathrm{lbs}$, and $600 \mathrm{lbs}$ boxes of cargo, would now only lift the $400 \mathrm{lbs}$ and $600 \mathrm{lbs}$ boxes of cargo due to the elimination of the $200 \mathrm{lbs}$ box.

\subsection{Literature Review}

A review of research concerning failure time distribution parameters impacted by loading and life conditions have revealed that concentrating on failure time or cycles-to-failure alone might not be a correct or informative approach to the problem. The conventional method is to create a life distribution from field or test data. Alternatively, Wang et al (1) implements a failure model that specifically takes load applications as a parameter. They approach this by applying randomized loads to a component. This cyclic load is then classified as either a load that weakens the strength or has no effect. The respective reliabilities of the components are then calculated under both circumstances laying the foundation for a failure rate model.

Wang et al (2) takes into account the frequency of loading in failure distributions. There are other similar papers dealing with system reliability and failure rate modeling such as He et al (3), and Cazuguel et al (4).

\section{COMPONENT RELIABILITY MODELS CONSIDERING STRESS CYCLE DISTRIBUITION}

\subsection{Baseline Model Without Load Adjustment}

A system consists of many constituent components that can fail. These parts are the components. For every component failure data should be recorded and with this failure data different methods can be applied if there are varying stresses associated with each cycle. A basic method records all stress cycles regardless of any stress or loading metric and provides a baseline model. It provides the same results as the conventional method of calculating a failure cycle distribution. The other four methods require varying amounts of data and can be useful for different situations.

Four different methods are presented to estimate future reliability given discrete cycles that are variable and shifting in the future. The different models depend on the quality and quantity of the data that is obtained. Selection between the four methods to use is largely dependent on the available data and if stresses are linear or nonlinearly related to the component. Assuming that a particular system or component are impacted by each stress cycle similarly, and that stress variations do not cause any notable differences, a simple counting of the stress cycles where,

$$
t_{s}=\sum_{i} t_{i}
$$

$t_{s}=$ total number stress cycles

$t_{i}=$ total number stress cycles of $i^{\text {th }}$ type

This is the conventional way to record cyclic failure data; there is no metric or measurement of stress or load associated with the cycle count. The weakness of this method is that it cannot account for a future stress profile, as stress is not even considered. The lack of stress cycles and profiles in this traditional method proves to be disadvantageous for trying to predict reliability with a changing stress profile on a system.

\subsection{Linear Load Adjusted Stress Cycles}

In many cases a small stress produces a smaller contribution to component failure as opposed to a larger stress. To model this case where the stress impacts the reliability of the components, the force, tension or any usage stress metric unit is adjusted accordingly so that some stress cycles are counted (or weighted) more than others, as indicated in the following equation.

$$
t_{s}=\sum_{i} t_{i} \frac{s_{i}}{s_{\mathrm{o}}}
$$

$t_{s}=$ number of stress adjusted usage cycles

$s_{i}=$ stress for the $i^{\text {th }}$ stress type

$s_{\mathrm{o}}=$ baseline stress

The baseline metric unit can be some average stress value over some time interval or a force associated with the most common type of load. All reliability analysis and models can be conducted using the adjusted number of usage cycles $\left(t_{s}\right)$ and not the original cycle count. 
This method provides a ratio to scale the impact of the loads and organizes the impact into numerical values based on some average impact metric unit. This is done by keeping the baseline $\left(s_{0}\right)$ the same, $s_{i}$, is shifted to accommodate the future usage stress profile. This is done for every $t_{i}$ until the user meets the desired composition of stresses. Once the shift is quantified, the linear adjusted $t_{s}$ is used as the life metric to estimate Weibull distribution parameters using the concepts of maximum likelihood estimation (MLE).

The advantages in creating an adjusted load count, is by having a model that can reflect the relative stresses associated with each cycle. The adjusted load count produces a model where the usage stress variables can increase or decrease more quickly over a shifting stress distribution. Some of the disadvantages are that the model depends on a linear assumption which may or may not be the case. If stresses effect the component in a nonlinear matter another method maybe needed.

\subsection{Non-Linear Load Adjusted Stress Cycles}

The second model is mathematically similar to the first but it recognizes that some component types are very sensitive to increased stress while others may not be affected by the stress at all. The non-linear stress adjusted $t_{s}$ contains all the aspects of the linear model; however the exponential $a_{j}$ provides more sensitivity to specific stress conditions. If the stress level has no effect on the component, $a_{j}$ can be set to 0 and only the numbers of stress cycles on the component are considered. The exponent enables the load to adjust to the appropriate amount of stress on the subsystem or component.

$$
t_{s}=\sum_{i} t_{i}\left(\frac{s_{i}}{s_{\mathrm{o}}}\right)^{a_{j}}
$$

$a_{j}=$ nonlinear adjustment factor for component $j$

An example of using the $a_{j}$ parameter set to zero might be if the system contains an electrical component exposed to a mechanical load. This component would not be dependent on the stress level and thus simply counting $t_{i}$ is sufficient

Determining the $a_{j}$ parameter is based on how the load and stresses are related. It might be difficult to determine this value for all components. Also, the equation is only indirectly sensitive to the stress increases on the component.

\subsection{Models as a Function of Load Profiles}

Method 3 requires more data as well as a meaningful stress metric unit. The components are exposed to changing stress profiles, and therefore, for every cycle-to-failure, there is an associated mean and standard deviation for stress for each cycle-to-failure. Considering the crane example, the crane lifts a combination of 200, 400 and $600 \mathrm{lbs}$ boxes of cargo, and each cycle-to-failure would have a mean and standard deviation attached to when the crane failed. The composition of cargo would be different for every single failure time as the usage until failure would be different for every failure. By taking the mean and standard deviation, a Weibull parameter can be calculated to represent the failure distribution. The Weibull scale parameter, $\eta$, becomes a function of the mean and standard deviation of stresses, and $\eta$ shifts into a more appropriate value to represent the future stress profile, as indicated in the following equations.

$$
\begin{aligned}
f(t)= & \frac{\beta}{\eta}\left(\frac{t}{\eta}\right)^{\beta-1} e^{-\left(\frac{t}{\eta}\right)^{\beta}}, t>0, \beta>0, \eta>0 \lambda(t) \\
& =\frac{\beta}{\eta}\left(\frac{t}{\eta}\right)^{\beta-1} \\
\eta \quad & \eta_{\mathrm{o}} \exp \left(-b_{1} \mu-b_{2} \sigma\right)
\end{aligned}
$$

$f(t)=$ probability density function for cycles-to-failure

$\beta=$ Weibull shape parameter

$\eta \quad=$ Weibull scale parameter

$\eta_{\mathrm{o}}=$ baseline Weibull scale parameter

$t=$ number of usage cycles

$\mu \quad=$ average stress

$\sigma=$ standard deviation of stresses

$b_{1}=$ parameter coefficient for mean stress

$b_{2}=$ parameter coefficient for standard deviation

All of the model parameters can be estimated given an appropriate data set considering the mean and standard deviation as accelerated stress levels in an accelerated life model. The MLE can be used for this purpose.

An increasing stress distribution can be considered by adjusting the mean and standard deviation to the anticipated future levels. As the mean and standard deviation increase the exponential function decreases, this causes $\eta$ to decrease. Thus increasing and decreasing $\eta$ causes an increase and decrease of the time to failure.

The advantages of this method are that the impact of a stress or force can be directly monitored and can distinguish the impact of stress on a component. The disadvantage would be that a large and comprehensive data set is needed. Also large variability can significantly affect results and in some cases the mean and standard deviation might not be sufficient enough in describing the different load profiles.

\subsection{Analysis based on Load Profile (Relative Frequency)}

This final model provides the most robust parameters by organizing the failure data into subcategories. Once these subcategories are determined, a percentage or proportion number is given to each subcategory or in this case $x_{i}$. Every cycle-to-failure is associated with specific stress values that can be distributed into the defined categories. Any number of categories can exist and the load may increase or decrease reliability by the simple calculation of changing $x_{i}$ into either a positive or negative value. This method provides the most organized conditions as well as directly relates the increased force, or stress on the subcomponent. However, the data must be comprehensive and excess variability impacts results. This method also requires more failure data points than the other methods.

In this method, the Weibull scale parameter is a modeled as mathematical function of the relative frequency of usage cycles with more stress associated with them. The equation is as follows. 


$$
\begin{aligned}
& \quad f(t)=\frac{\beta}{\eta}\left(\frac{t}{\eta}\right)^{\beta-1} e^{-\left(\frac{t}{\eta}\right)^{\beta}}, t>0, \beta>0, \eta>0 \lambda(t) \\
& =\frac{\beta}{\eta}\left(\frac{t}{\eta}\right)^{\beta-1} \\
& \eta=\eta_{0} \exp \left(b_{1} x_{1}+b_{2} x_{2}+b_{3} x_{3}+\cdots\right) \\
& \sum_{i} x_{i}=1 \\
& x_{1}=1^{\text {st }} \text { relative frequency } \\
& x_{2}=2^{\text {nd }} \text { relative frequency }
\end{aligned}
$$

$\sum_{i} x_{i}=1$

$x_{1}=1^{\text {st }}$ relative frequency

$x_{3}=3^{\text {rd }}$ relative frequency

All of the model parameters can be estimated given an appropriate data set, and as in method 3, the parameters can be estimated using accelerated stress levels in an accelerated life model using MLE. As it is difficult to calculate the likelihood function for these parameters, the use of software designed for this purpose is recommended.

\section{COMPONENT EXAMPLES}

\subsection{Description of Examples}

This paper is motivated by an actual application with real data but the data collection is a work in progress. For demonstration purposes, simulated data is used. The cycles-tofailure data (or CTF) was generated by simulation and serves as the basis in explaining the four methods. In this example there are three categories of stress for the usage cycles with varying degrees of force or stress. The three categories are separated by 200-500 lbs., 500-700 lbs., and 700-1000 lbs. For this example these are the critical values as to which the stress on the system is evaluated.

\subsection{Method 1: Linear Adjusted Stress Cycles}

In the linear model all that is needed are the CTF data and the actual stress level for each cycle. Once the data is gathered such as in Table 1, a baseline stress of $s_{0}$ must be determined. In this case a simple value of $650 \mathrm{lbs}$. was chosen.

Table 1 Results For Linear Adjusted Count

\begin{tabular}{|r|r|r|}
\hline 2 & CTF & Linear Adjusted Count \\
\hline 3 & 553.95 & 472.09 \\
\hline 4 & 705.21 & 765.11 \\
\hline 5 & 1237.65 & 2356.57 \\
\hline 6 & 564.06 & 489.49 \\
\hline 7 & 472.10 & 342.89 \\
\hline 8 & 430.15 & 284.66 \\
\hline 9 & 61.90 & 5.89 \\
\hline 10 & 541.15 & 450.53 \\
\hline 11 & 846.15 & 1101.50 \\
\hline 12 & 719.05 & 795.43 \\
\hline 13 & 1050.78 & 1698.67 \\
\hline 14 & 295.21 & 134.08 \\
\hline 15 & 209.48 & 67.51 \\
\hline 16 & 547.47 & 461.11 \\
\hline 17 & 481.27 & 356.34 \\
\hline 18 & 410.80 & 259.62 \\
\hline 19 & 219.80 & 74.32 \\
\hline 20 & 204.50 & 64.34 \\
\hline 21 & 185.22 & 52.78 \\
\hline 22 & 355.18 & 194.08 \\
\hline 23 & 188.90 & 54.90 \\
\hline & & \\
\hline
\end{tabular}

The 650 lbs. represents the common stress during the duration of the data collection. Using Method 1, all stresses are divided by the baseline stress. In essence the CTF data is being modified to account for more or less stress cycles by utilizing a ratio. Table 1 also depicts the adjusted CTF, and although only about 20 points are shown, Table 1 only represents a third of the data used for Figure 2. Using the adjusted CTF data a Weibull distribution is formed with a $\beta$ of 3.3 and $\eta$ of 433 cycles.

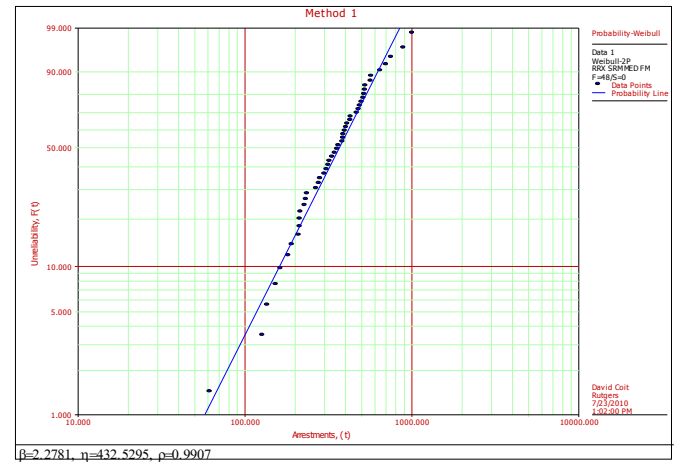

Figure 2 Weibull Plot for Linear Adjusted Count

In Method 1, each cycle is adjusted and thus the CTF is adjusted as well. Once these new CTF are plotted and the MLE is then used to calculate the future stress profiles for a Weibull distribution.

\subsection{Nonlinear Adjusted Stress Cycles}

The nonlinear adjusted method is mathematically similar to the linear adjusted method. The ratio of $\left(s_{i} / s_{\mathrm{o}}\right)$ or stress

\begin{tabular}{|c|c|c|c|}
\hline 2 & CTF & Nonlinear Adjusted Count & $a_{j}=1.5$ \\
\hline 3 & 670.46 & 702.364397 & \\
\hline 4 & 736.69 & 736.6932777 & \\
\hline 5 & 550.82 & 550.815023 & \\
\hline 6 & 471.86 & 471.860959 & \\
\hline 7 & $1,020.23$ & 1020.232653 & \\
\hline 8 & 470.48 & 470.4786203 & \\
\hline 9 & 432.99 & 432.992592 & \\
\hline 10 & 366.38 & 366.3788394 & \\
\hline 11 & 856.73 & 856.7278651 & \\
\hline 12 & 942.26 & 942.2555909 & \\
\hline 13 & 731.84 & 731.8363705 & \\
\hline 14 & 661.21 & 661.2130578 & \\
\hline 15 & 484.94 & 484.9402926 & \\
\hline 16 & 602.60 & 602.6012368 & \\
\hline 17 & 778.77 & 778.7703375 & \\
\hline 18 & 703.07 & 703.0675283 & \\
\hline 19 & 979.51 & 979.5113658 & \\
\hline 20 & $1,267.59$ & 1267.588752 & \\
\hline 21 & 837.14 & 837.1385951 & \\
\hline 22 & 491.26 & 491.2645147 & \\
\hline 23 & $1,049.52$ & 1049.524329 & \\
\hline
\end{tabular}
over the baseline stress ratio adjusts the cycles. However the difference between the linear and the nonlinear count is the $a_{j}$

\section{Table 2 Results for Nonlinear Adjusted Count}

parameter. When the nonlinear adjusted number is utilized, the $a_{j}$ parameter will change the linearity by making, $t_{s}$ an exponential function. Table 2 shows 23 points out of 50 using the $a_{j}$ parameter and how the adjusted count was calculated. 


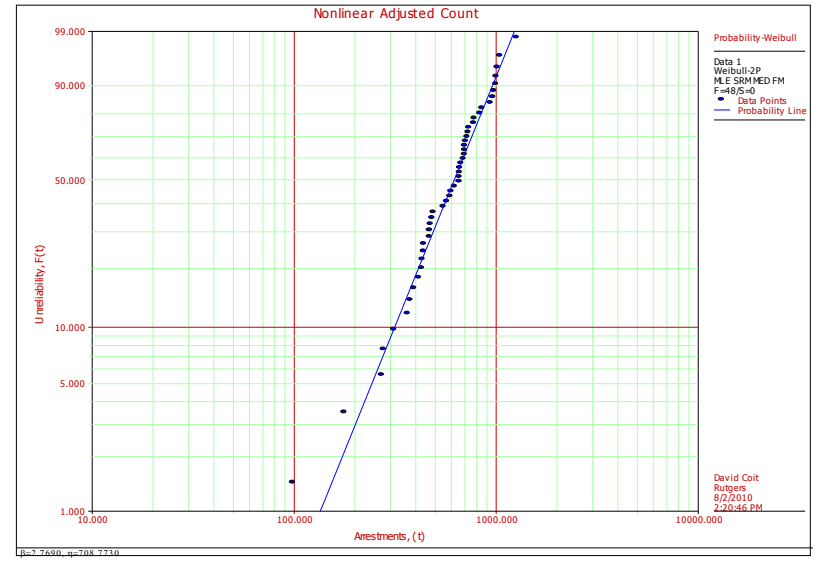

Figure 3 Weibull Plot for Non Linear Adjusted Count

When the adjusted nonlinear failure count is taken into consideration, the maximum likelihood estimator produces a $\beta$ of 2.25 and a $\eta$ of 465 as shown in Figure 2. The user must define the $a_{j}$ parameter and in some occasions, it can be problematic to select it. In this example, $a_{j}$ was equal to 1.5.

\subsection{Weibull Model as a Function of Load Profiles}

Method 3 and 4 are mathematically different from the linear and nonlinear methods and require calculating a $\eta$ based upon the corresponding stress conditions set by the data. Both methods can be utilized to project future compositions of stress to calculate reliability. Method 3 concentrates on acquiring the mean and standard deviation stress, while Method 4 relies on the relative frequency or composition of the stress. Sample data for both Method 3 and 4 are presented in Table 3. Method 3 utilized the mean and standard deviation of the stress for each usage cycle until failure. This means that every cycle has an associated stress. Method 3 incorporates the mean and standard deviations of stress exposure and parameters $b_{1}$ and $b_{2}$ are estimated using the MLE using the general log-linear model for accelerated life testing. Table 3 shows a sample of data that is used to determine the results in Figure 3.

Each CTF has a stress mean and standard deviation depicted in Table 3. To estimate reliability for an anticipated stress profiles, an anticipated future mean and standard deviation would be input into the $\eta$ equation and the life parameter becomes a function of the future mean and standard deviation.

As mentioned previously, Method 3 requires more data than Methods 1 and 2; however the benefit of this model is that heavier loads will directly impact each CTF. The results for the example are $b_{1}=-0.0038$ and $b_{2}=-0.0014, \beta=2.51$ and $\eta_{0}=4712$ cycles. $\eta_{0}$ is the initial value while $\eta$ is the adjusted parameter. The coefficient $b_{2}$ causes the Weibull scale parameter to decrease as the standard deviation increases, while the $b_{1}$ coefficient causes the scale parameter to decrease with increased mean stress.
Table 3 Relative Frequency, Mean, and Standard Deviation Calculations

\begin{tabular}{|c|c|c|c|c|c|}
\hline & $200-500$ & $500-700$ & $700-1000$ & & \\
\hline Stress Cycle & $x 1$ & $x 2$ & x3 & $E[X]$ & $\mathrm{SD}[\mathrm{X}]$ \\
\hline 184.40 & 0.26 & 0.34 & 0.40 & 635.98 & 200.22 \\
\hline 320.75 & 0.20 & 0.10 & 0.70 & 723.16 & 202.31 \\
\hline 377.97 & 0.41 & 0.18 & 0.41 & 599.88 & 226.54 \\
\hline 170.54 & 0.12 & 0.24 & 0.64 & 730.69 & 174.35 \\
\hline 540.10 & 0.68 & 0.12 & 0.20 & 478.50 & 200.03 \\
\hline 141.91 & 0.16 & 0.34 & 0.50 & 685.75 & 183.29 \\
\hline 369.52 & 0.67 & 0.03 & 0.30 & 508.51 & 228.69 \\
\hline 464.11 & 0.49 & 0.16 & 0.35 & 564.98 & 227.00 \\
\hline 345.17 & 0.58 & 0.20 & 0.22 & 510.43 & 204.21 \\
\hline 508.85 & 0.93 & 0.07 & 0.00 & 368.56 & 69.01 \\
\hline 218.48 & 0.08 & 0.49 & 0.43 & 686.98 & 156.23 \\
\hline 490.29 & 0.54 & 0.12 & 0.34 & 550.03 & 229.07 \\
\hline 340.41 & 0.64 & 0.23 & 0.12 & 470.41 & 176.51 \\
\hline 453.90 & 0.55 & 0.07 & 0.38 & 557.37 & 236.77 \\
\hline 274.77 & 0.29 & 0.45 & 0.26 & 591.32 & 184.72 \\
\hline 438.34 & 0.34 & 0.42 & 0.23 & 571.38 & 187.44 \\
\hline 495.03 & 0.77 & 0.12 & 0.11 & 436.31 & 168.33 \\
\hline 549.69 & 0.61 & 0.22 & 0.18 & 493.57 & 194.17 \\
\hline 404.42 & 0.85 & 0.02 & 0.13 & 421.31 & 171.44 \\
\hline 612.96 & 0.53 & 0.38 & 0.09 & 491.02 & 164.82 \\
\hline 328.41 & 0.22 & 0.74 & 0.04 & 555.95 & 120.66 \\
\hline 182.65 & 0.09 & 0.35 & 0.57 & 719.30 & 163.08 \\
\hline 913.67 & 0.93 & 0.06 & 0.01 & 370.53 & 77.86 \\
\hline 267.40 & 0.44 & 0.33 & 0.23 & 547.59 & 198.11 \\
\hline 113.72 & 0.73 & 0.01 & 0.27 & 484.78 & 220.98 \\
\hline 74.31 & 0.58 & 0.33 & 0.10 & 480.06 & 166.64 \\
\hline 764.79 & 0.71 & 0.19 & 0.10 & 447.18 & 164.62 \\
\hline 76.77 & 0.44 & 0.14 & 0.42 & 594.60 & 231.48 \\
\hline 146.85 & 0.13 & 0.38 & 0.49 & 688.65 & 175.30 \\
\hline 147.29 & 0.13 & 0.41 & 0.47 & 685.36 & 172.44 \\
\hline 400.14 & 0.32 & 0.63 & 0.05 & 531.11 & 134.59 \\
\hline
\end{tabular}

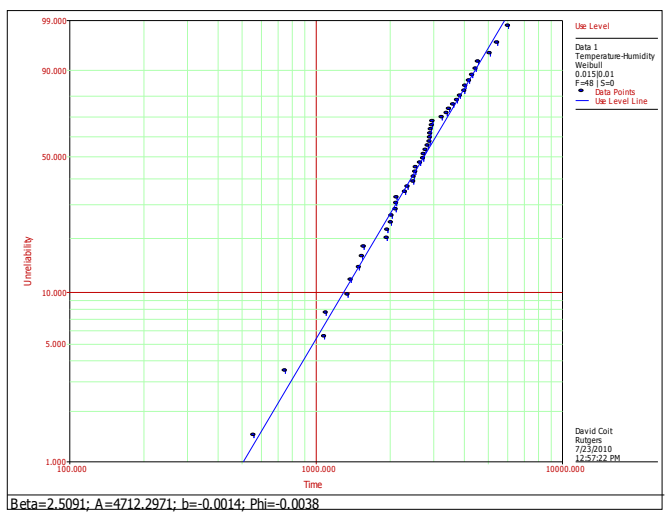

Figure 4 Method 3 Mean and Standard Deviation of Adjusted Cycle Weibull Plot

\subsection{Weibull Model as a Function of Load Profiles (Relative}

Frequency)

Method 4 operates under the same principal as Method 3 but instead of the mean and standard deviation, the relative frequencies of the stresses are used to categorize stress into three different ranges. The stresses are divided into proportions and are represented by the variables $x_{1}, x_{2}$ and $x_{3}$. These relative frequencies must add up to one and can be divided even further, but for this example only three 
categories were used.

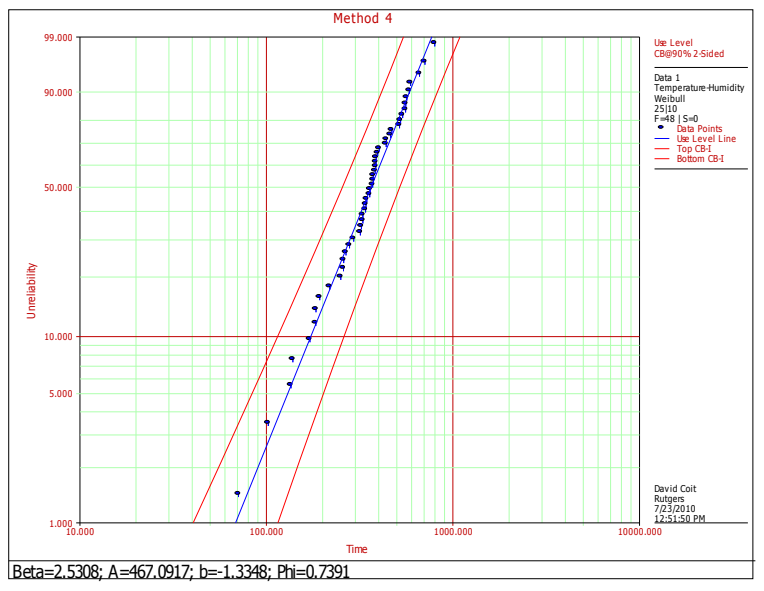

Figure 5 Weibull Plot for Relative Frequency Method

The MLE uses the relative frequencies of each stress CTF and computes an associated coefficient $b_{i}$. In this example, $b_{2}$ is equal to 0 , which means that stresses in the most central category do not increase or decrease $\eta$. The variables $b_{1}$ and $b_{3}$ are calculated using the MLE using software, and a log-linear model. The Weibull plot is shown in Figure 5.

In Table 3 the relative frequencies as well as the CTFs are presented and the data indicates that $b_{1}$ and $b_{3}$ are equal to 0.7 and -1.3 respectively. $\eta_{0}$ is 467 cycles and $\beta$ is equal to 2.53 .

Choosing a composition of only middle ranked stress cycles $\left(x_{1}=0, x_{2}=1, x_{3}=0\right)$ produces the same mean as a mixed set $\left(x_{1}=0.2, x_{2}=0.6, x_{3}=0.2\right)$; however this method may likely produce a lower $\eta$ for the more diverse frequency set. Method 4 penalizes the heavier stresses and causes the $\eta$ to decrease, due to a negative exponential function. The lighter stress cycles cause $\eta$ to increase; thus increasing life.

\section{SYSTEM EXAMPLE}

The purpose of this paper is to describe and demonstrate methods to predict reliability for future stress profiles with current data. The tool used for the analysis will is discrete event simulation. Figure 6 represents a reliability block diagram of a system in which Method 4 had been used to estimate future reliability. The first component utilized an $\eta_{0}$ of 617 cycles and a $\beta_{0}$ of 2.59 this comes from the calculation of Method 4 of a $\beta$ of 2.59 and parameters $b_{1}, b_{2}$, and $b_{3}$

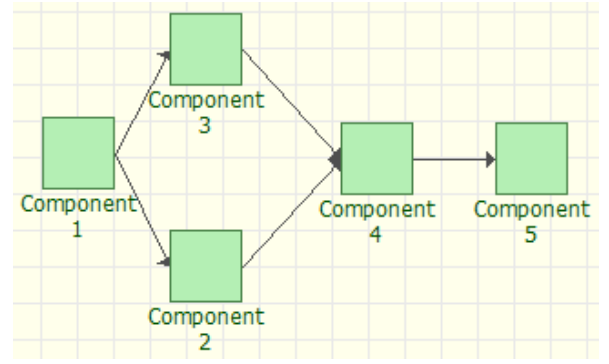

Figure 6 Reliability Block Diagram

equaling $0.3,0$, and -1.5 . This is just a simple example of the utilization of the methods developed at a system level.

\section{CONCLUSIONS}

To predict system reliability corresponding to a future stress profile using existing failure data is a difficult task. There are many factors to consider such as the accuracy of the data given and the amount. High failure data items are able to utilize Method 3 and Method 4. However this is not always possible and thus alternatives like Methods 1 and 2 may be utilized. Although not mathematically intensive, Methods 1 and 2 provide a viable option that can work reasonably well with variable and scarce data.

\section{REFERENCES}

1. Wang, Zheng, Kang, Rui and Liyang, Xi. Modeling A Failure Rate Model with the Number of Load application as a Life Parameter. China Mechanical Engineering, 2009-2010.

2. Wang, Zheng, Li-yang, Xie and Bing, Li. Reliability Model of Failure-Dependent System with Frequency of Loading Taken into Account. Journal of Northeastern University, 2007.

3. Jian. He, Lin Cheng,Yuanzhang Sun. Analysis of Components' Reliability Modeling Based On Real-time Operating Conditions International Conference on Power System Technology, 2006.

4. M. Cazuguel, C. Renaud, J. Y. Cognard,.Time-variant Reliability of Nonlinear Structures: Application to a Representative Part of a Plate Floor. Quality and Reliability Engineering International, 2006, Vol. 22.

5. Zhang, Xiang and Ernst, Gockenbach. Component Reliability Modeling of Distribution Systems Based on the Evaluation of Failure Statistics. IEEE Transactions on Dielectrics and Electrical Insulation, 2007, Vol. 14.

\section{BIOGRAPHIES}

Akira Hada

Department of Industrial Engineering, Rutgers University 96 Frelinghuysen Road, Piscataway, NJ 08854 USA

e-mail: akhada@rutgers.edu

Akira Hada is an MS at Rutgers University where he also received his $\mathrm{BS}$ degree in industrial engineering. For the past year he has worked with NAVAIR in the development of reliability models to support the recovery gear service life analysis program.

David W. Coit, $P h D$

Department of Industrial Engineering, Rutgers University

96 Frelinghuysen Road, Piscataway, NJ 08854 USA

e-mail: coit@rutgers.edu

David W. Coit is a Professor in the Department of Industrial engineering at Rutgers University. He received a BS degree in mechanical engineering from Cornell University, and MBA from Rensselaer Polytechnic Institute, and MS \& Ph.D. in industrial engineering from the University of Pittsburgh. He also has over 10 years of experience working for ITT 
Research Institute (ITTRI), Rome, NY. He is a member of IIE and INFORMS.

\section{Mark Agnello}

Naval Air Engineering Station Lakehurst

Lakehurst, New Jersey 08733

e-mail: mark.agnello1@navy.mil

Mark Agnello graduated from New Jersey Institute of Technology in 1987 and received a BSEE. He worked for the Naval Air Propulsion Center, Trenton, NJ from 1987-1994 as an instrumentation and control systems engineer in gas turbine engine test cells. In 1994 he transferred to the Naval Air Systems Command (NAVAIR), Patuxent River, MD. Mr. Agnello now works at the Naval Support Activity, Lakehurst, $\mathrm{NJ}$ as a team leader in the R\&M engineering group supporting aircraft launch and recovery equipment (ALRE).

Keith Megow

Naval Air Engineering Station Lakehurst

Lakehurst, New Jersey 08733

e-mail: keith.megow@navy.mil

Keith Megow graduated from Rutgers University in 1983 with a BSME and New Jersey Institute of Technology in 2002 with an MSEE. He worked for the Naval Air Propulsion Center, Trenton, NJ from 1983-1997 as a test engineer. Then from 1997 to 2008 he worked at Fort Monmouth in the area of signals intelligence. From 2008 to present he has worked at the Naval Air Warfare Center, Lakehurst, NJ in the field of reliability and maintainability for a variety of aircraft launch and recovery programs. 